\title{
Spin pumping at terahertz nutation resonances
}

\author{
Ritwik Mondal $\odot^{1,2, *}$ and Akashdeep Kamra $\odot^{3, \dagger}$ \\ ${ }^{1}$ Department of Spintronics and Nanoelectronics, Institute of Physics of the Czech Academy of Sciences, Cukrovarnická 10, \\ CZ - 16200 Praha 6, Czech Republic \\ ${ }^{2}$ Department of Physics and Astronomy, Uppsala University, Box 516, Uppsala, SE-75120, Sweden \\ ${ }^{3}$ Condensed Matter Physics Center (IFIMAC) and Departamento de Física Teórica de la Materia Condensada, \\ Universidad Autónoma de Madrid, E-28049 Madrid, Spain
}

(Received 11 October 2021; revised 2 December 2021; accepted 9 December 2021; published 20 December 2021)

\begin{abstract}
We investigate spin pumping current injected by the nutation resonances of a ferromagnet (FM) or an antiferromagnet (AFM) into an adjacent metal. Comparing the dc spin pumping current between the normal precession and the nutation resonances, we find that the ratio of spin pumping current at the nutation resonance to the precession resonance is more pronounced in AFMs. We further show that the spin pumping current injected by the nutation resonance is opposite in sign as compared with the normal precession mode. This could offer a useful experimental signature for identifying such nutation resonances. Analyzing the nature of the nutational eigenmodes, we show that the sign change in spin current is rooted in a reversal of the precession sense for the nutation mode(s). Furthermore, the nutational modes in AFMs are found to be dominated by precession of one of the two sublattices only.
\end{abstract}

DOI: 10.1103/PhysRevB.104.214426

\section{INTRODUCTION}

The ultrafast manipulation of spins at the terahertz frequencies has paramount scientific and technological interest. Most spin dynamics experiments have been explained so far by the traditional Landau-Lifshitz-Gilbert (LLG) phenomenology [1-7], which describes a precessional motion of spins around an effective field and an energy dissipation via a viscous damping term $[8,9]$. However, at ultrafast time scales, the LLG equation has been found to be insufficient $[10,11]$.

To address this shortcoming, the LLG phenomenology has been extended to account for magnetization dynamics in the inertial regime $[12,13]$. Essentially, the inclusion of magnetic inertia leads to a spin nutation at the ultrashort time scales and can be incorporated via a double-time derivative of the magnetization, i.e., $\boldsymbol{M} \times \ddot{\boldsymbol{M}}[14,15]$. The inertial LLG (ILLG) equation of motion for two sublattice systems has the form

$$
\dot{\boldsymbol{M}}_{i}=-\gamma_{i}\left(\boldsymbol{M}_{i} \times \boldsymbol{H}_{i}^{\mathrm{eff}}\right)+\frac{\boldsymbol{M}_{i}}{\boldsymbol{M}_{i 0}} \times\left[\alpha_{i} \dot{\boldsymbol{M}}_{i}+\eta_{i} \ddot{\boldsymbol{M}}_{i}\right],
$$

with the gyromagnetic ratio $\gamma$, the effective field $\boldsymbol{H}^{\text {eff }}$, the ground-state magnetic moment $M_{0}$, the Gilbert damping parameter $\alpha$, and the inertial relaxation time $\eta$. The index " $i$ "

\footnotetext{
*mondal@fzu.cz

†akashdeep.kamra@uam.es
}

Published by the American Physical Society under the terms of the Creative Commons Attribution 4.0 International license. Further distribution of this work must maintain attribution to the author(s) and the published article's title, journal citation, and DOI. Funded by Bibsam. denotes the sublattice. In general, Gilbert damping in a two sublattice and the inertial relaxation time are tensors [16-20]. Here we treat them as scalars for simplicity and in an attempt to capture the leading-order effects. The emergence of spin nutation has been attributed to several proposed mechanisms [21-28]. The characteristic time scales of the nutation $\eta$ have been predicted to be in a range of $1-100$ fs $[29,30]$. Experimentally inertial relaxation time $\eta$ is found to be about hundreds fs in two sublattice FMs [31].

The spin nutation additionally introduces a second resonance in the FM resonance (FMR) spectrum, however, at a higher frequency in $\mathrm{THz}$ range. Such a resonance is called FM nutation resonance (FMNR) [32,33]. Moreover, the precession resonance frequencies are decreased due to the spin nutation [30,32,34]. In a more recent experiment, several higher-order nutation resonances have been observed [35]. With rapid recent progress, new pathways for the role of nutation in practical devices have already started to emerge [36].

Despite several signatures in ultrafast spin dynamics experiments attributed to nutation modes [29,31,35], further smoking gun validations are needed. Complementary to a time-resolved tracking of the magnetization dynamics, the spin pumping current $[37,38]$ injected by the latter into an adjacent metal has emerged as a powerful probe. It has already been employed in investigating a broad range of phenomena from the spin Seebeck effect [39-44] to AFM resonance [45-47] and over a broad range of time scales. With the anticipation that spin pumping current driven by the nutation mode might be very different from the conventional resonance modes, we theoretically investigate this hypothesis in both FMs and AFMs here finding valuable results and insights. 
In this article, we theoretically investigate the dc spin pumping current injected by a magnet driven by an oscillating magnetic field into an adjacent metal. We consider both FMs and AFMs and focus on their nutation modes. We find that the spin current at the nutation resonance is negative, while the spin current at the precession resonance is positive. Our results also show that the spin current at the nutation resonance increases with increasing inertial relaxation time $\eta$ in FM and AFM. The computed ratio of spin currents at the nutation to the precession resonance shows that the nutation spin current is more pronounced in AFM. We delineate the nutation eigenmodes finding the FM nutation to entail magnetization precession in the opposite sense compared with the normal precession mode. In AFM, the nutation modes are characterized by a larger precession cone for one of the two sublattice magnetizations, thereby departing from the nearly collinear dynamics in the conventional AFM resonance. These features may enable a clear distinction of the nutation modes in experiments.

We now comment on the potential significance of higherorder spin torques in describing spin dynamics. In comparison to Eq. (1), the third- and fourth-order spin torques have the following forms $\zeta(\boldsymbol{M} \times \dddot{\boldsymbol{M}})$ and $\delta(\boldsymbol{M} \times \dddot{\boldsymbol{M}})$, respectively (the sublattice index $i$ has been omitted). Such perturbative expansion entails a progressive smallness of the higher-order terms making them relevant at increasingly higher frequencies. Presently, estimates for the corresponding coefficients $\zeta$ and $\delta$ are not known. At THz frequencies, the second-order spin torque term is enough to describe the spin dynamics and be fully consistent with the experimental observations. Therefore we restrict ourselves to Eq. (1) and analyze the precession and nutation resonances within the linear-response theory.

\section{SPIN PUMPING IN FMs}

We consider a FM with a single sublattice as $\boldsymbol{M}=M_{0} \hat{z}$ at the ground state that is under the influence of an external Zeeman field $\boldsymbol{H}=H_{0} \hat{\boldsymbol{z}}$. Such a FM can be described by the following free-energy $\mathcal{F}(\boldsymbol{M})=-H_{0} M_{z}-K M_{z}^{2} / M_{0}^{2}$, where $K$ is the uniaxial anisotropy energy and $M_{0}$ is the ground-state magnetic moment. The effective field that enters into the ILLG equation can thus be calculated using $\boldsymbol{H}^{\text {eff }}=$ $-\partial \mathcal{F} / \partial \boldsymbol{M}$.

When a small oscillating transversal field $\boldsymbol{h}(t)=$ $h_{x}(t) \hat{\boldsymbol{x}}+h_{y}(t) \hat{\boldsymbol{y}}$ is applied, the small magnetization oscillations are induced such that time-dependent magnetization is $\boldsymbol{M}(t)=m_{x}(t) \hat{\boldsymbol{x}}+m_{y}(t) \hat{\boldsymbol{y}}+M_{z}(t) \hat{\boldsymbol{z}}$, where $M_{z}(t)=\sqrt{M_{0}^{2}-m_{x}^{2}(t)-m_{y}^{2}(t)} \approx M_{0}$. Within the linear-response theory in the circular basis described by $h_{ \pm}=h_{x} \pm i h_{y}=h e^{ \pm i \omega t}$ and $m_{ \pm}=m_{x} \pm i m_{y}=m e^{ \pm i \omega t}$, the calculated susceptibility expression is [48]

$$
m_{ \pm}=\frac{\gamma M_{0}}{\Omega_{0}-\eta \omega^{2}-\omega \pm i \omega \alpha} h_{ \pm}=\chi_{ \pm} h_{ \pm},
$$

where $\Omega_{0}=\frac{\gamma}{M_{0}}\left[H_{0} M_{0}+2 K\right]$. The poles of the susceptibility determine the resonance frequencies. Without the nutation term $\eta$, only a FMR frequency is obtained. However, the spin

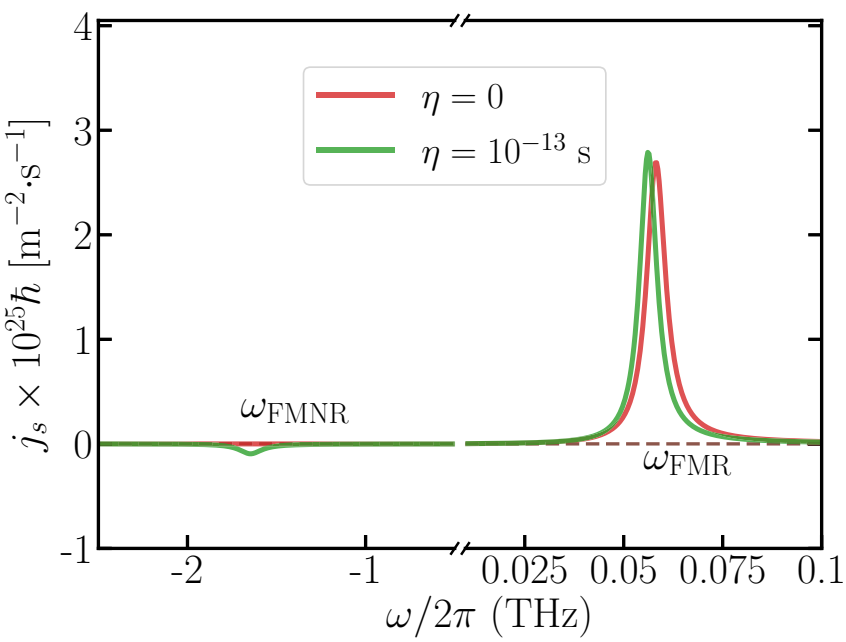

FIG. 1. The calculated spin pumping dc current for inertial relaxation times $\eta=0 \mathrm{~s}$ and $\eta=10^{-13} \mathrm{~s}$. The used parameters are $M_{0}=$ $2 \mu_{B}, K=10^{-23} \mathrm{~J}, \gamma=1.76 \times 10^{11} \mathrm{~T}^{-1} \cdot \mathrm{s}^{-1}, \alpha=0.05, H_{0}=1 \mathrm{~T}$, $|h|=10^{-3} \mathrm{~T}, g_{r}^{\uparrow \downarrow}=10^{19} \mathrm{~m}^{-2}$.

nutation additionally introduces a second resonance FMNR frequency. These two frequencies are [48]

$$
\begin{aligned}
\omega_{\mathrm{FMR}} & =\frac{\sqrt{1+4 \eta \Omega_{0}}-1}{2 \eta}, \\
\omega_{\mathrm{FMNR}} & =-\frac{\sqrt{1+4 \eta \Omega_{0}}+1}{2 \eta} .
\end{aligned}
$$

The negative frequency in the nutation resonance dictates the fact that the nutation resonance has opposite handedness of rotation compared with the FMR [48,49]. This has been further corroborated by an analysis of the nutation eigenmode presented in Appendix A.

The dc component of a generated spin current density can be expressed as $[37,50]$

$$
j_{s}=\frac{\omega}{2 \pi} \int_{0}^{2 \pi / \omega} \frac{\hbar}{4 \pi} g_{r}^{\uparrow \downarrow} \frac{1}{M_{0}^{2}}[\boldsymbol{M}(t) \times \dot{\boldsymbol{M}}(t)]_{z} d t .
$$

We calculate the spin current in the circular basis and $[\boldsymbol{M}(t) \times$ $\dot{\boldsymbol{M}}(t)]_{z}=\frac{i}{2}\left[m_{+} \dot{m}_{-}-m_{-} \dot{m}_{+}\right]$. Using Eq. (2), the calculated spin current has the expression

$$
j_{s}=\frac{\hbar}{4 \pi} g_{r}^{\uparrow \downarrow}\left[\frac{\omega \gamma^{2}}{\left(\Omega_{0}-\eta \omega^{2}-\omega\right)^{2}+\alpha^{2} \omega^{2}}|h|^{2}\right] .
$$

Figure 1 displays the spin current computation results obtained without and with inertial relaxation time $\eta=10^{-13} \mathrm{~s}$. We denote the calculated spin current at FMR frequencies as $j_{s}^{\mathrm{FMR}}$ and at nutation frequencies as $j_{s}^{\mathrm{FMNR}}$. Note that the spin current at the FMR frequencies has a positive sign; however, the calculated spin current at the nutation resonance frequencies has the opposite sign. The reason is that while the precession mode rotates anticlockwise, the nutation mode rotates clockwise.

Nonetheless, we calculate the ratio of spin currents calculated at several inertial relaxation times $\eta$ in Fig. 2. Note that the ratio of spin currents is independent of several parameters including spin mixing conductance $g_{r}^{\uparrow \downarrow}$, small field $h$, etc. We emphasize that the spin current at the nutation resonance 


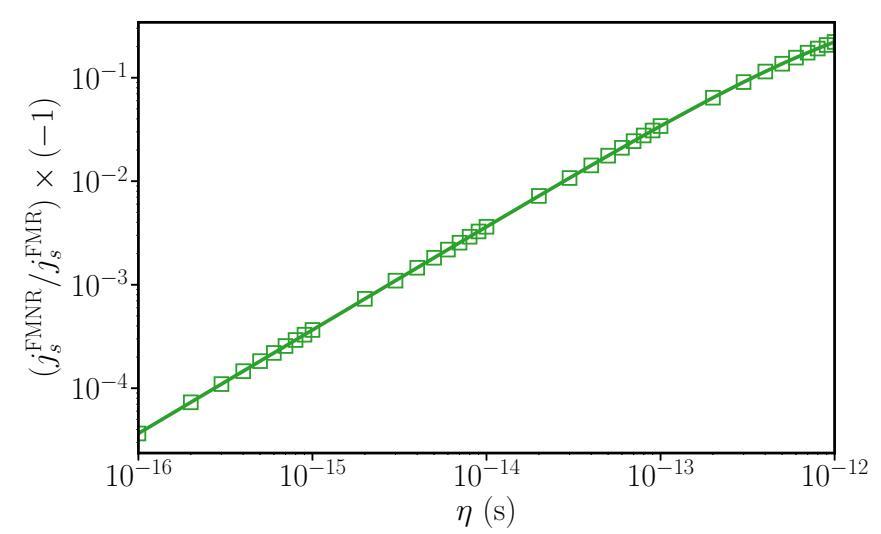

FIG. 2. The ratio of spin current for FMs at the nutation resonance to the precession resonance vs inertial relaxation time $\eta$. The used parameters are $M_{0}=2 \mu_{B}, \gamma=1.76 \times 10^{11} \mathrm{~T}^{-1} \cdot \mathrm{s}^{-1}, \alpha=0.05$, $K=10^{-23} \mathrm{~J}$, and $H_{0}=0 \mathrm{~T}$.

increases linearly with $\eta$, while the current at the precession resonance stays almost constant. Such an observation can easily be understood from Eq. (6). The FMR frequency lies in the $\mathrm{GHz}$ regime and its shift due to nutation is small in FMs. Therefore, the calculated spin current at the FMR

$$
\left(\begin{array}{l}
h_{A \pm} \\
h_{B \pm}
\end{array}\right)=\left(\begin{array}{c}
\frac{1}{\gamma_{A} M_{A 0}}\left(\Omega_{A} \pm i \omega \alpha_{A}-\eta_{A} \omega^{2}-\omega\right) \\
\frac{J}{M_{A 0} M_{B 0}}
\end{array}\right.
$$

with the following definitions $\Omega_{A}=\frac{\gamma_{A}}{M_{A 0}}\left(J+2 K_{A}+H_{0} M_{A 0}\right)$, $\Omega_{B}=\frac{\gamma_{B}}{M_{B 0}}\left(J+2 K_{B}-H_{0} M_{B 0}\right)$. For an AFM, we consider, $\gamma_{A}=\gamma_{B}=\gamma, M_{A 0}=M_{B 0}=M_{0}, K_{A}=K_{B}=K, \alpha_{A}=\alpha_{B}=$ $\alpha, \eta_{A}=\eta_{B}=\eta$. With the assumption that $J \gg H_{0} M_{0}$ and $J \gg K$, we have $\Omega_{A} \approx \Omega_{B} \approx \gamma J / M_{0}$. Therefore, the obtained approximate frequencies of the antiferromagnetic precession resonance (AFMR) and antiferromagnetic nutation resonance $(\mathrm{AFMNR})$ are $[48,51]$

$$
\begin{gathered}
\omega_{\mathrm{AFMR}} \approx \pm \frac{\gamma}{M_{0}} \sqrt{\frac{4 J K}{1+\frac{2 \eta \gamma J}{M_{0}}}}, \\
\omega_{\mathrm{AFMNR}} \approx \pm \frac{\sqrt{1+\frac{2 \eta \gamma J}{M_{0}}}}{\eta} .
\end{gathered}
$$

Note that unlike FM, the AFM has two sublattices meaning there are two precession resonance frequencies and corresponding two nutation frequencies. However, we also mention that in a two sublattice FM there are two of each precession and nutation resonance frequencies, e.g., $\mathrm{CoFeB}[31,34]$.

First we examine and compare the conventional and nutational eigenmodes in an AFM. To this end, we set is roughly proportional to $\omega_{\mathrm{FMR}}^{-1} \approx 1 / \Omega_{0}+\eta$. However, the dominant spin current contribution at the FMNR is $\omega_{\text {FMNR }}^{-1} \approx$ $-\eta$. Therefore, at smaller $\eta$, the ratio of spin currents is linear in $\eta$; however, the ratio deviates from linearity at larger $\eta$.

\section{SPIN PUMPING IN AFM}

We consider an AFM having two sublattices, namely, $A$ and $B$. The ground state of such AFM is $\boldsymbol{M}_{A}=M_{A 0} \hat{z}$ and $\boldsymbol{M}_{B}=$ $-M_{B 0} \hat{z}$ under an influence of external applied Zeeman field $\boldsymbol{H}=H_{0} \hat{z}$. The free energy of the system $[18,48]$

$$
\begin{aligned}
\mathcal{F}\left(\boldsymbol{M}_{A}, \boldsymbol{M}_{B}\right)= & -H_{0}\left(M_{A z}+M_{B z}\right) \\
& -\frac{K_{A}}{M_{A 0}^{2}} M_{A z}^{2}-\frac{K_{B}}{M_{B 0}^{2}} M_{B z}^{2}+\frac{J}{M_{A 0} M_{B 0}} \boldsymbol{M}_{A} \cdot \boldsymbol{M}_{B},
\end{aligned}
$$

containing the Zeeman field, uniaxial anisotropy energies for individual sublattices in terms of $K_{A}$ and $K_{B}$, and the intersublattice magnetic exchange energy $J$. The effective field in the ILLG equation can be calculated using the following definition: $\boldsymbol{H}_{A}^{\text {eff }}=-\partial \mathcal{F} / \partial \boldsymbol{M}_{A}$ and $\boldsymbol{H}_{B}^{\text {eff }}=-\partial \mathcal{F} / \partial \boldsymbol{M}_{B}$. Similar to our consideration of FM, we calculate the AFM susceptibility assuming $\boldsymbol{h}_{A}=\boldsymbol{h}_{B}$ and $\boldsymbol{M}_{A}(t)=M_{A 0} \hat{z}+\boldsymbol{m}_{A}(t)$ and $\boldsymbol{M}_{B}(t)=$ $-M_{B 0} \hat{z}+\boldsymbol{m}_{B}(t)$. In the circular basis, the inverse susceptibility expression for AFM is obtained as [48]

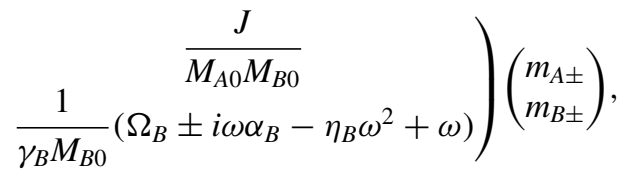

$h_{A \pm}=h_{B \pm}=0$ and $\alpha=0$ in Eq. (8) and obtain two following equation for $m_{A+}$ and $m_{B+}$ :

$$
\begin{aligned}
& \left(\Omega_{A}-\eta \omega^{2}-\omega\right) m_{A+}+\frac{\gamma J}{M_{0}} m_{B+}=0, \\
& \left(\Omega_{B}-\eta \omega^{2}+\omega\right) m_{B+}+\frac{\gamma J}{M_{0}} m_{A+}=0 .
\end{aligned}
$$

Two of such similar equation can also be obtained for $m_{A-}$ and $m_{B-}$. At AFMR, we drop the nutation term and the ratio between $m_{A+}$ and $m_{B+}$ is obtained as

$$
\frac{m_{A+}}{m_{B+}}=-\frac{\frac{\gamma J}{M_{0}}}{\Omega_{A}-\omega_{\mathrm{AFMR}}} \approx-\frac{1}{1-\frac{\omega_{\mathrm{AFMR}} M_{0}}{\gamma J}} .
$$

One can easily calculate that $\frac{\omega_{\mathrm{AFMR}} M_{0}}{\gamma^{J}} \ll 1$ and can be neglected. Therefore, we obtain $m_{A+} \approx-m_{B+}$ implying that the two sublattice magnetizations remain nearly antiparallel subtending almost equal cone angles $\theta_{A} \approx \theta_{B}$ in AFMR, as depicted in Fig. 3 (left panel).

For nutation mode, we similarly obtain the ratio with the leading term in AFMNR frequency as $\omega_{\text {AFMNR }} \approx 1 / \eta$ as

$$
\frac{m_{A+}}{m_{B+}}=-\frac{\frac{\gamma J}{M_{0}}}{\Omega_{A}-\eta \omega_{\mathrm{AFMNR}}^{2}-\omega_{\mathrm{AFMNR}}}=\frac{1}{\frac{2 M_{0}}{\eta \gamma J}-1} .
$$




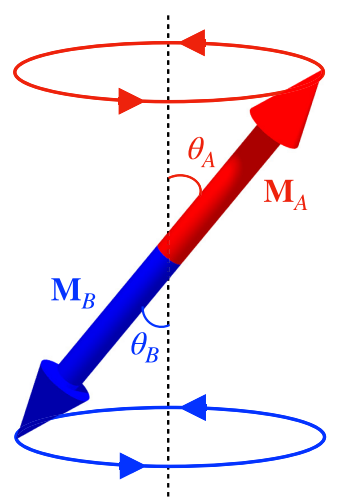

Precession Modes

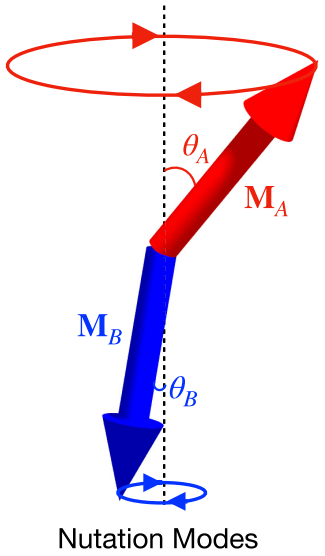

FIG. 3. The precession and nutation modes in AFMs. The precession modes behave as normal AFMs, but for the nutation mode, the spins are not antiparallel giving rise to faster dynamics in one sublattice, while the dynamics is not experienced by the other sublattice.

Employing $\frac{M_{0}}{\eta \gamma J} \gg 1$, we find $m_{A+} \ll m_{B+}$. This means at the AFMNR, the two sublattices do not align exactly antiparallel, giving rise to large cone angle in one sublattice, while the other sublattice precesses with a much smaller cone angle. These characteristics of the nutation eigenmodes have been shown in Fig. 3 (right panel).

Next we calculate the spin pumping current following Refs. [52,53]. To this end, we consider the effects of intrasublattice and also cross-sublattice terms in the spin pumping current. The spin mixing conductance for an AFM interfaces with a metal is a $2 \times 2$ tensor and depends sensitively on the interface [52,54]. A disordered interface (expected to be common) effectively behaves as an uncompensated interface [52,54]. Here we report the contributions due to the intra-sublattice and cross-sublattice spin pumping separately, keeping in mind that the final result is a weighted sum of the two where the weight depends on the interface [52,54]. The intra-sublattice contributions can be calculated as $\boldsymbol{M}_{A}(t) \times$ $\dot{\boldsymbol{M}}_{A}(t)+\boldsymbol{M}_{B}(t) \times \dot{\boldsymbol{M}}_{B}(t)=\omega\left(m_{A+} m_{A-}+m_{B+} m_{B-}\right)$ and the cross-sublattice contributions as $\boldsymbol{M}_{A}(t) \times \dot{\boldsymbol{M}}_{B}(t)+\boldsymbol{M}_{B}(t) \times$
$\dot{\boldsymbol{M}}_{A}(t)=\omega\left(m_{A+} m_{B-}+m_{B+} m_{A-}\right)$. The computed spin current due to intra- and cross-sublattice contributions become

$$
\begin{aligned}
& j_{s, \text { Intra }}=\frac{\hbar}{4 \pi} g_{r}^{\uparrow \downarrow} \times \omega\left(\frac{m_{A-} m_{A+}}{M_{A 0}^{2}}+\frac{m_{B-} m_{B+}}{M_{B 0}^{2}}\right) \\
& j_{s, \text { Cross }}=\frac{\hbar}{4 \pi} g_{r}^{\uparrow \downarrow} \times \omega\left(\frac{m_{A-} m_{B+}}{M_{A 0} M_{B 0}}+\frac{m_{B-} m_{A+}}{M_{B 0} M_{A 0}}\right) .
\end{aligned}
$$

The total spin current is calculated from both the intra- and cross-sublattice contributions as

$$
j_{s, \text { Total }}=j_{s, \text { Intra }}+j_{s, \text { Cross }} .
$$

The computed spin currents are shown for intra-, cross-, and total sublattice terms at the inertial relaxation time $\eta=100 \mathrm{fs}$ in Fig. 4. Without the application of a static magnetic field, the two AFM precession resonance modes have exactly the same frequency, however, opposite in sign. Therefore, the spin currents appear at the resonance frequencies. Due to the nutation resonance, additional spin current contributions can be observed at the higher $\mathrm{THz}$ nutation frequencies. Following the results of FM case, the sign of nutation spin currents will be opposite to the corresponding spin current at the precession resonance mode for intra-sublattice contributions.

As observed, the magnitude of spin currents at the nutation resonances is small compared with the spin currents at the precession resonance at the lower $\eta$, e.g., 1 fs. However, it increases rapidly for higher $\eta$ and surpasses the spin currents at the precession resonance already at $\eta \sim 10 \mathrm{fs}$. Such observation in AFMs is in contrast to the FM, where the nutation spin current is smaller even at $\eta=1 \mathrm{ps}$. Note that the AFM precession resonance is suppressed and thus the spin current at the precession resonance is smaller compared with that of the FM resonance.

For intra-sublattice contributions, the nutation spin current has opposite sign to the precession spin current, consistent with our findings for FMs [see Fig. 4(a)]. However, for the cross-sublattice contributions, the precession spin current changes sign, while the nutation spin current does not change sign compared with the intra-sublattice contributions [see Fig. 4(b)]. This is due to the fact that the two sublattices in the AFM precession mode remain almost antiparallel to each other. On the other hand, for the nutation modes in AFM, the two sublattices do not align antiparallel to each other and
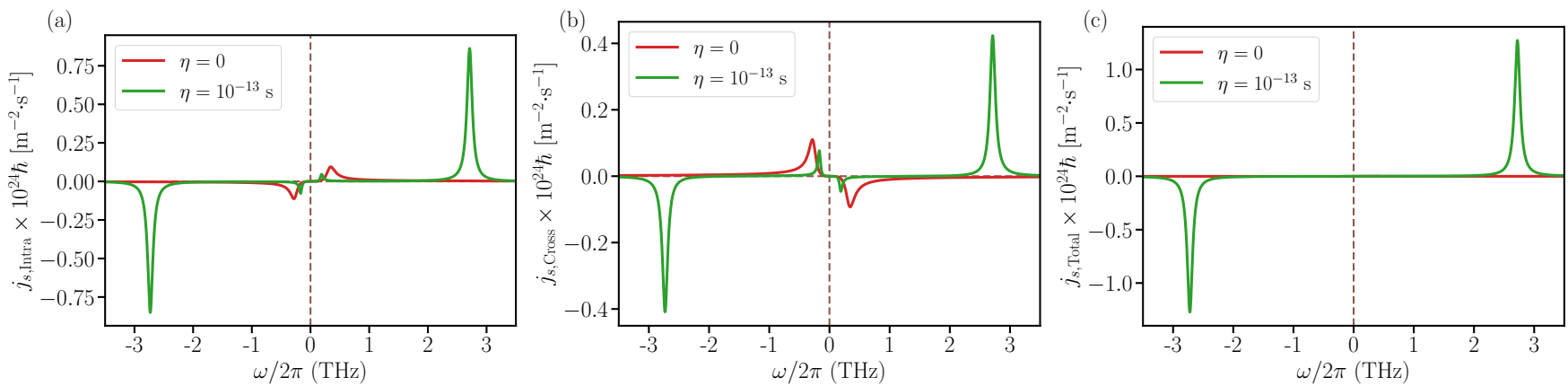

FIG. 4. The spin current contributions for (a) intra-sublattice terms: $\boldsymbol{M}_{A}(t) \times \dot{\boldsymbol{M}}_{A}(t)+\boldsymbol{M}_{B}(t) \times \dot{\boldsymbol{M}}_{B}(t)$, (b) cross-sublattice terms: $\boldsymbol{M}_{A}(t) \times$ $\dot{\boldsymbol{M}}_{B}(t)+\boldsymbol{M}_{B}(t) \times \dot{\boldsymbol{M}}_{A}(t)$, and (c) total with both intra- and cross-sublattice terms calculated without and with the inertial relaxation time i.e. $\eta=0$ and $\eta=10^{-13} \mathrm{~s}$. The used parameters are: $M_{A 0}=M_{B 0}=M_{0}=2 \mu_{B}, \gamma_{A}=\gamma_{B}=\gamma=1.76 \times 10^{11} \mathrm{~T}^{-1} \cdot \mathrm{s}^{-1}, \hbar=1.05 \times 10^{-34} \mathrm{~m}^{2} \cdot \mathrm{kg} \cdot \mathrm{s}^{-1}$, $g_{r}^{\uparrow \downarrow}=10^{-19} \mathrm{~m}^{-2}, H_{0}=0 \mathrm{~T}, h_{A}=h_{B}=10^{-3} \mathrm{~T}, K_{A}=K_{B}=K=10^{-23} \mathrm{~J}, J=10^{-21} \mathrm{~J}, \alpha_{A}=\alpha_{B}=\alpha=0.05$, and $\eta_{A}=\eta_{B}=\eta$. 


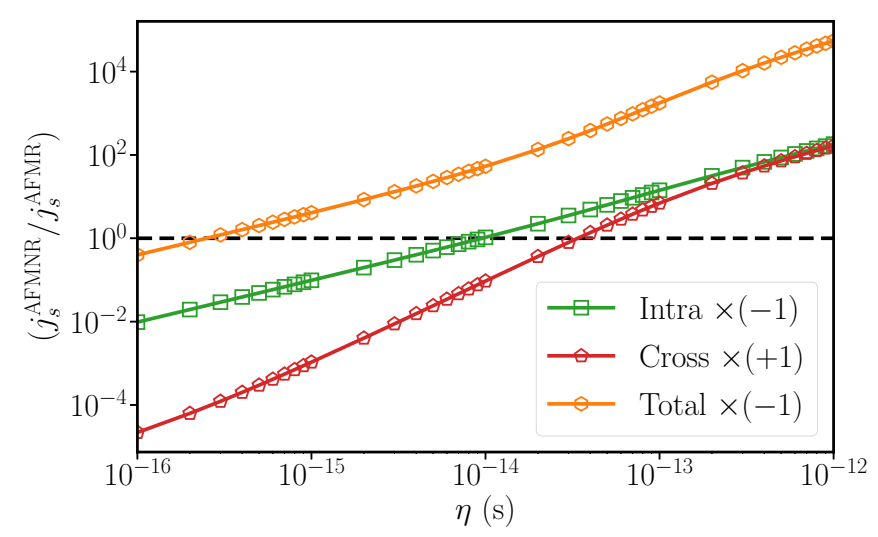

FIG. 5. The ratio of spin current for AFMs at the nutation resonance to the precession resonance vs inertial relaxation time. The used parameters are: $M_{A 0}=M_{B 0}=M_{0}=2 \mu_{B}, \gamma_{A}=\gamma_{B}=$ $\gamma=1.76 \times 10^{11} \mathrm{~T}^{-1} \cdot \mathrm{s}^{-1}, H_{0}=0 \mathrm{~T}, K_{A}=K_{B}=K=10^{-23} \mathrm{~J}, J=$ $10^{-21} \mathrm{~J}, \alpha_{A}=\alpha_{B}=\alpha=0.05$, and $\eta_{A}=\eta_{B}=\eta$.

hence the nutation spin current does not change sign for intraand cross-sublattice contribution. Due to such properties, the total spin currents at the precession resonance almost cancel with each other; however, the total nutation spin currents add up [see Fig. 4(c)], assuming equal intra- and cross-sublattice spin mixing conductances. Therefore, the total spin currents are enhanced at the nutation resonances, while those are suppressed at the precession resonances.

To understand such enhancement of spin currents, we compute the ratio of spin currents at the nutation resonance to the precession resonance in Fig. 5. By doing so, the results are independent of several parameters used in obtaining Fig. 4. For example, the ratio of spin currents is independent of spin mixing conductance $g_{r}^{\uparrow \downarrow}$, small field $h$, etc. Compared with the FM spin current in Fig. 1, such ratio already crosses unity within $\eta=10 \mathrm{fs}$ for AFM. As explained earlier, due to the cancellation of the spin current at the precession resonance, the ratio for total contribution increases rapidly. In fact, the total spin current at the nutation resonance is already higher even below $\eta=1$ fs.

\section{CONCLUSIONS}

To conclude, we present a theoretical investigation of spin pumping with the magnetic inertial dynamics for one and two sublattice systems. The magnetic inertial dynamics additionally introduces a spin pumping current at the $\mathrm{THz}$ nutation resonance frequencies. However, due to the opposite sense of rotation in precession and nutation modes, the spin pumping current has an opposite sign at the nutation resonance compared with the one at precession resonance. Such scenario remains the same for intra-sublattice spin current in AFM, while the cross-sublattice spin current only changes sign at the precession resonance. Thus the spin current ratio is negative and positive for AFM intra- and cross-sublattice terms, respectively. While the two sublattices remain almost antiparallel in AFM at the precessional resonance, the antiparallel alignment is broken at the nutational resonance resulting in a large cone angle in one of the sublattices. Our obtained results should motivate the experimental search of magnetic inertial dynamics in magnets via detection of spin pumping currents.

\section{ACKNOWLEDGMENTS}

We acknowledge the Swedish Research Council (VR Grant No. 2019-06313) and the Spanish Ministry for Science and Innovation-AEI Grant No. CEX2018-000805-M (through the "Maria de Maeztu" Programme for Units of Excellence in $R \& D)$.

\section{APPENDIX: THE CHARACTERISTICS OF NUTATION RESONANCE IN FM}

Here we investigate the characteristics of precession and nutation resonance modes in FM. Following Ref. [48], we write the equation for inverse susceptibility as in Eq. (B6) of Ref. [48],

$$
\begin{aligned}
\left(\begin{array}{l}
h_{x} \\
h_{y}
\end{array}\right)= & \frac{1}{\gamma M_{0}}\left(\begin{array}{cc}
\Omega_{0}+\alpha \frac{\partial}{\partial t}+\eta \frac{\partial^{2}}{\partial t^{2}} & -\frac{\partial}{\partial t} \\
\frac{\partial}{\partial t} & \Omega_{0}+\alpha \frac{\partial}{\partial t}+\eta \frac{\partial^{2}}{\partial t^{2}}
\end{array}\right) \\
& \times\left(\begin{array}{l}
m_{x} \\
m_{y}
\end{array}\right) .
\end{aligned}
$$

We replace $m_{x}=m_{y} \propto e^{i \omega t}$ such that $\frac{\partial}{\partial t} \rightarrow i \omega$ and $\frac{\partial^{2}}{\partial t^{2}} \rightarrow$ $-\omega^{2}$. Therefore, Eq. (A1) can be recast as

$\left(\begin{array}{l}h_{x} \\ h_{y}\end{array}\right)=\frac{1}{\gamma M_{0}}\left(\begin{array}{cc}\Omega_{0}+i \alpha \omega-\eta \omega^{2} & -i \omega \\ i \omega & \Omega_{0}+i \alpha \omega-\eta \omega^{2}\end{array}\right)\left(\begin{array}{l}m_{x} \\ m_{y}\end{array}\right)$.

Now we employ $h_{x}=h_{y}=0$ and $\alpha=0$ in order to examine the nature of the eigenmodes, finding a relation between $m_{x}$ and $m_{y}$. We obtain two following equations:

$$
\begin{aligned}
\left(\Omega_{0}-\eta \omega^{2}\right) m_{x}-i \omega m_{y} & =0 \\
i \omega m_{x}+\left(\Omega_{0}-\eta \omega^{2}\right) m_{y} & =0 .
\end{aligned}
$$

The Eqs. (A3) and (A4) can be written in concise forms as

$$
\begin{gathered}
m_{x}=\frac{i \omega}{\Omega_{0}-\eta \omega^{2}} m_{y} \\
m_{y}=-\frac{i \omega}{\Omega_{0}-\eta \omega^{2}} m_{x} .
\end{gathered}
$$

The precession and nutation resonance frequencies that were obtained previously in Ref. [48] are:

$$
\begin{gathered}
\omega_{\mathrm{FMR}}=\frac{\sqrt{1+4 \eta \Omega_{0}}-1}{2 \eta} \approx \Omega_{0}-\eta \Omega_{0}^{2} \\
\omega_{\mathrm{FMNR}}=-\frac{\sqrt{1+4 \eta \Omega_{0}}+1}{2 \eta} \approx-\frac{1}{\eta}-\Omega_{0}\left(1-\eta \Omega_{0}\right) .
\end{gathered}
$$

We replace the leading order precession resonance term i.e. $\omega_{\mathrm{FMR}} \approx \Omega_{0}$ in Eqs. (A5) and (A6). Therefore, at the precession resonance we find the relation between $m_{x}$ and $m_{y}$ as 


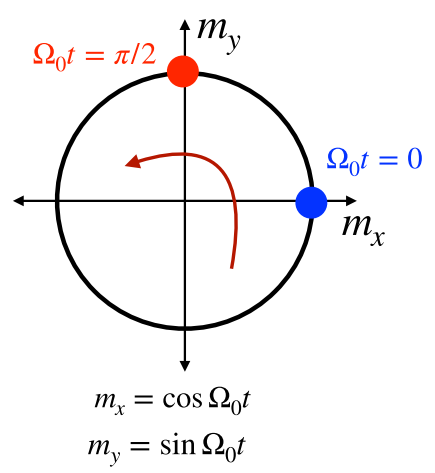

Precession mode

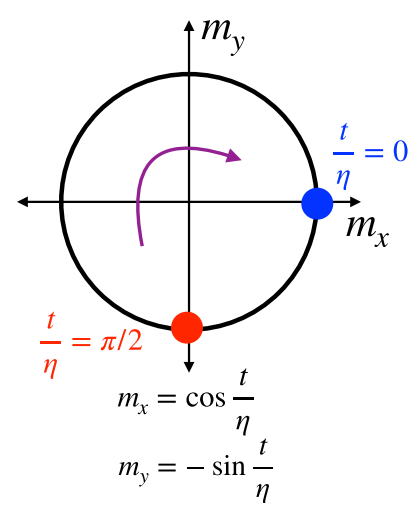

Nutation mode
FIG. 6. The precession and nutation mode in FM. Notice that the precession and nutation modes have opposite sense of rotation.

follows

$$
\begin{gathered}
m_{x}=\frac{i \Omega_{0}}{\Omega_{0}} m_{y}=i m_{y} \\
m_{y}=-\frac{i \Omega_{0}}{\Omega_{0}} m_{x}=-i m_{x}
\end{gathered}
$$

However, the obtained $m_{x}$ and $m_{y}$ are complex quantities. In order to obtain the real and time-dependent parts at the precession resonance, we compute:

$$
\begin{gathered}
m_{x}=\operatorname{Re}\left[m_{x} e^{i \omega t}\right]=\operatorname{Re}\left[m_{x} e^{i \Omega_{0} t}\right]=m_{x} \cos \Omega_{0} t \\
m_{y}=\operatorname{Re}\left[m_{y} e^{i \omega t}\right]=\operatorname{Re}\left[-i m_{x} e^{i \Omega_{0} t}\right]=m_{x} \sin \Omega_{0} t .
\end{gathered}
$$

At the nutation resonance, however, we replace the leadingorder frequency term, i.e., $\omega_{\mathrm{FMNR}} \approx-1 / \eta$ and find

$$
\begin{gathered}
m_{x}=\frac{i(-1 / \eta)}{\Omega_{0}-1 / \eta} m_{y}=-\frac{i}{\eta \Omega_{0}-1} m_{y}=i m_{y} \\
m_{y}=-\frac{i(-1 / \eta)}{\Omega_{0}-1 / \eta} m_{x}=\frac{i}{\eta \Omega_{0}-1} m_{x}=-i m_{x} .
\end{gathered}
$$

We again calculate the real and time-dependent parts at the nutation resonance as

$$
\begin{gathered}
m_{x}=\operatorname{Re}\left[m_{x} e^{i \omega t}\right]=\operatorname{Re}\left[m_{x} e^{-i \frac{t}{\eta}}\right]=m_{x} \cos \frac{t}{\eta} \\
m_{y}=\operatorname{Re}\left[m_{y} e^{i \omega t}\right]=\operatorname{Re}\left[-i m_{x} e^{-i \frac{t}{\eta}}\right]=-m_{x} \sin \frac{t}{\eta} .
\end{gathered}
$$

Such characteristics of precession and nutation resonance have been shown in Fig. 6 .
[1] E. Beaurepaire, J.-C. Merle, A. Daunois, and J.-Y. Bigot, Phys. Rev. Lett. 76, 4250 (1996).

[2] T. Kampfrath, M. Battiato, P. Maldonado, G. Eilers, J. Notzold, S. Mahrlein, V. Zbarsky, F. Freimuth, Y. Mokrousov, S. Blügel, M. Wolf, I. Radu, P. M. Oppeneer, and M. Münzenberg, Nat. Nano. 8, 256 (2013).

[3] J. Walowski and M. Münzenberg, J. Appl. Phys. 120, 140901 (2016).

[4] R. John, M. Berritta, D. Hinzke, C. Müller, T. Santos, H. Ulrichs, P. Nieves, J. Walowski, R. Mondal, O. ChubykaloFesenko, J. McCord, P. M. Oppeneer, U. Nowak, and M. Münzenberg, Sci. Rep. 7, 4114 (2017).

[5] T. A. Ostler, J. Barker, R. F. L. Evans, R. W. Chantrell, U. Atxitia, O. Chubykalo-Fesenko, S. El Moussaoui, L. Le Guyader, E. Mengotti, L. J. Heyderman, F. Nolting, A. Tsukamoto, A. Itoh, D. Afanasiev, B. A. Ivanov, A. M. Kalashnikova, K. Vahaplar, J. Mentink, A. Kirilyuk, Th. Rasing et al., Nat. Commun. 3, 666 (2012).

[6] S. Wienholdt, D. Hinzke, K. Carva, P. M. Oppeneer, and U. Nowak, Phys. Rev. B 88, 020406(R) (2013).

[7] S. Wienholdt, D. Hinzke, and U. Nowak, Phys. Rev. Lett. 108, 247207 (2012).

[8] L. D. Landau and E. M. Lifshitz, Phys. Z. Sowjetunion 8, 153 (1935) [Ukr. J. Phys. 53, 14 (2008)].

[9] T. L. Gilbert, IEEE Trans. Magn. 40, 3443 (2004).

[10] T. G. H. Blank, K. A. Grishunin, E. A. Mashkovich, M. V. Logunov, A. K. Zvezdin, and A. V. Kimel, Phys. Rev. Lett. 127, 037203 (2021).
[11] R. Mondal, A. Donges, U. Ritzmann, P. M. Oppeneer, and U. Nowak, Phys. Rev. B 100, 060409(R) (2019).

[12] M.-C. Ciornei, J. M. Rubí, and J.-E. Wegrowe, Phys. Rev. B 83, 020410(R) (2011).

[13] J.-E. Wegrowe and M.-C. Ciornei, Am. J. Phys. 80, 607 (2012).

[14] H. Suhl, IEEE Trans. Magn. 34, 1834 (1998).

[15] D. Böttcher and J. Henk, Phys. Rev. B 86, 020404(R) (2012).

[16] R. Mondal, M. Berritta, and P. M. Oppeneer, Phys. Rev. B 94, 144419 (2016).

[17] R. Mondal, M. Berritta, and P. M. Oppeneer, Phys. Rev. B 98, 214429 (2018).

[18] A. Kamra, R. E. Troncoso, W. Belzig, and A. Brataas, Phys. Rev. B 98, 184402 (2018).

[19] P. Nieves, U. Atxitia, R. W. Chantrell, and O. ChubykaloFesenko, Low Temp. Phys. 41, 739 (2015).

[20] H. Y. Yuan, Q. Liu, K. Xia, Z. Yuan, and X. R. Wang, Europhys. Lett. 126, 67006 (2019).

[21] M. Fähnle and C. Illg, J. Phys.: Condens. Matter 23, 493201 (2011).

[22] M. Fähnle, D. Steiauf, and C. Illg, Phys. Rev. B 84, 172403 (2011).

[23] S. Bhattacharjee, L. Nordström, and J. Fransson, Phys. Rev. Lett. 108, 057204 (2012).

[24] U. Bajpai and B. K. Nikolić, Phys. Rev. B 99, 134409 (2019).

[25] R. Mondal, M. Berritta, A. K. Nandy, and P. M. Oppeneer, Phys. Rev. B 96, 024425 (2017).

[26] R. Mondal, M. Berritta, and P. M. Oppeneer, J. Phys.: Condens. Matter 30, 265801 (2018). 
[27] S. Giordano and P.-M. Déjardin, Phys. Rev. B 102, 214406 (2020).

[28] S. V. Titov, W. T. Coffey, Y. P. Kalmykov, M. Zarifakis, and A. S. Titov, Phys. Rev. B 103, 144433 (2021).

[29] Y. Li, A.-L. Barra, S. Auffret, U. Ebels, and W. E. Bailey, Phys. Rev. B 92, 140413(R) (2015).

[30] I. Makhfudz, E. Olive, and S. Nicolis, Appl. Phys. Lett. 117, 132403 (2020).

[31] K. Neeraj, N. Awari, S. Kovalev, D. Polley, N. Zhou Hagström, S. S. P. K. Arekapudi, A. Semisalova, K. Lenz, B. Green, J.C. Deinert, I. Ilyakov, M. Chen, M. Bawatna, V. Scalera, M. d'Aquino, C. Serpico, O. Hellwig, J.-E. Wegrowe, M. Gensch, and S. Bonetti, Nat. Phys. 17, 245 (2021).

[32] E. Olive, Y. Lansac, M. Meyer, M. Hayoun, and J.-E. Wegrowe, J. Appl. Phys. 117, 213904 (2015).

[33] M. Cherkasskii, M. Farle, and A. Semisalova, Phys. Rev. B 102, 184432 (2020).

[34] R. Mondal, J. Phys.: Condens. Matter 33, 275804 (2021).

[35] V. Unikandanunni, R. Medapalli, M. Asa, E. Albisetti, D. Petti, R. Bertacco, E. E. Fullerton, and S. Bonetti, Inertial spin dynamics in epitaxial cobalt films, (2021), arXiv:2109.03076.

[36] R. Rahman and S. Bandyopadhyay, J. Phys.: Condens. Matter 33, 355801 (2021).

[37] Y. Tserkovnyak, A. Brataas, and G. E. W. Bauer, Phys. Rev. B 66, 224403 (2002).

[38] E. Saitoh, M. Ueda, H. Miyajima, and G. Tatara, Appl. Phys. Lett. 88, 182509 (2006).

[39] K. Uchida, S. Takahashi, K. Harii, J. Ieda, W. Koshibae, K. Ando, S. Maekawa, and E. Saitoh, Nature (London) 455, 778 (2008).

[40] G. E. W. Bauer, E. Saitoh, and B. J. van Wees, Nat. Mater. 11, 391 (2012).

[41] J. Barker and G. E. W. Bauer, Phys. Rev. Lett. 117, 217201 (2016).
[42] T. S. Seifert, S. Jaiswal, J. Barker, S. T. Weber, I. Razdolski, J. Cramer, O. Gueckstock, S. F. Maehrlein, L. Nadvornik, S. Watanabe, C. Ciccarelli, A. Melnikov, G. Jakob, M. Münzenberg, S. T. B. Goennenwein, G. Woltersdorf, B. Rethfeld, P. W. Brouwer, M. Wolf, M. Kläui et al., Nat. Commun. 9, 2899 (2018).

[43] D. Hirobe, M. Sato, T. Kawamata, Y. Shiomi, K. I. Uchida, R. Iguchi, Y. Koike, S. Maekawa, and E. Saitoh, Nat. Phys. 13, 30 (2017).

[44] M. Weiler, M. Althammer, M. Schreier, J. Lotze, M. Pernpeintner, S. Meyer, H. Huebl, R. Gross, A. Kamra, J. Xiao, Y.-T. Chen, H. J. Jiao, G. E. W. Bauer, and S. T. B. Goennenwein, Phys. Rev. Lett. 111, 176601 (2013).

[45] R. Cheng, J. Xiao, Q. Niu, and A. Brataas, Phys. Rev. Lett. 113, 057601 (2014).

[46] P. Vaidya, S. A. Morley, J. van Tol, Y. Liu, R. Cheng, A. Brataas, D. Lederman, and E. del Barco, Science 368, 160 (2020).

[47] J. Li, C. B. Wilson, R. Cheng, M. Lohmann, M. Kavand, W. Yuan, M. Aldosary, N. Agladze, P. Wei, M. S. Sherwin, and J. Shi, Nature (London) 578, 70 (2020).

[48] R. Mondal, S. Großenbach, L. Rózsa, and U. Nowak, Phys. Rev. B 103, 104404 (2021).

[49] T. Kikuchi and G. Tatara, Phys. Rev. B 92, 184410 (2015).

[50] K. Ando, S. Takahashi, J. Ieda, Y. Kajiwara, H. Nakayama, T. Yoshino, K. Harii, Y. Fujikawa, M. Matsuo, S. Maekawa, and E. Saitoh, J. Appl. Phys. 109, 103913 (2011).

[51] R. Mondal and P. M. Oppeneer, Phys. Rev. B 104, 104405 (2021).

[52] A. Kamra and W. Belzig, Phys. Rev. Lett. 119, 197201 (2017).

[53] Q. Liu, H. Y. Yuan, K. Xia, and Z. Yuan, Phys. Rev. Mater. 1, 061401(R) (2017).

[54] R. E. Troncoso, M. A. Lund, A. Brataas, and A. Kamra, Phys. Rev. B 103, 144422 (2021). 